\title{
Los Valores en Los Consumidores Millennials de la Ciudad de Saltillo, Coahuila
}

\author{
Dra. Adriana Méndez, Wong \\ Mtra. Edith Reyes Ruiz \\ Dr. Jesús Francisco Mellado Siller \\ Karina Lizbeth Rodriguez Ramirez
}

Universidad Autónoma de Coahuila, Mexico

Doi: 10.19044/esj.2017.v13n28p371 URL:http://dx.doi.org/10.19044/esj.2017.v13n28p371

\begin{abstract}
This study makes a descriptive analysis of the clients profiles that belong to the Y or Millenial generation in the city of Saltillo, Coahuila. The study was performed using the Values List Scale (LOV) attributed to Khale (Khale, Beatty, \& Homer, 1986) quoted by Irene Ramos Soler (2008). This scale was used for the investigation of market segmentation and in identifying target markets. It is a work that has to do with the field of marketing. This study however is considered as an exploratory, descriptive, quantitative, and transversal investigation. Using Pearson's chi square analysis and an answer percentage comparison with the IBM SPSS software, they show us that the more important values are self-completion and selfesteem. A stratification sample was used. It also uses age variable and it applies a questionnaire instrument to 261 people that belong to $\mathrm{Y}$ generation in Saltillo, Coahuila.
\end{abstract}

Keywords: Psychographic segmentation, Values, Values List Scale (LOV)

\section{Resumen}

En este trabajo se hace un estudio descriptivo sobre los perfiles de clientes pertenecientes a la generación Y / millenials de la ciudad de Saltillo, Coahuila. Utilizando la Escala de Lista de Valores (LOV) atribuido a Khale ( Kahle , Beatty, \& Homer, 1986) citado por Irene Ramos Soler (2008), dicha escala ha sido utilizada en investigaciones sobre segmentación de mercados y para identificar mercados meta. Es un trabajo del campo del Marketing, se le considera una investigación de tipo exploratorio, descriptivo, cuantitativo y transversal. Realizando un análisis de ji cuadrado de Pearson y un comparativo en porcentajes de las respuestas con el programa de IBM SPSS los cuales nos muestran, que los valores mas 
importantes para los jóvenes consumidores entrevistados es la Autorrealización y la Autoestima. Se utiliza muestreo por estratificación, utilizando la variable edad, aplicando un Instrumento a 261 sujetos pertenecientes a la generación Y de la ciudad de Saltillo, Coahuila.

Palabras Claves: Segmentación psicográfica, Valores, Escala Lista de Valores (LOV)

\section{Introducción}

En los últimos años la investigación del consumidor ha ido en aumento. La lista de valores (LOV) es un método desarrollado por el centro de investigación de encuestas de la Universidad de Michigan y tiene su origen en los estudios sobre valores realizados por Maslow, Rokeach y Feather (Ramos Soler, 2008).

En este estudio se entrevistaron a 1,000 personas de la ciudad de Saltillo, Coahuila, determinando que 261 pertenecen a la "Generación Y" de acuerdo a la clasificación planteada Kotler y Keller (2012) en su libro "Dirección de Marketing" A continuación se presenta la revisión de la literatura con base al cual se plantean los objetivos, metodología y resultados obtenidos:

\section{Revisión de Literatura}

El hacer una buena segmentación nos garantiza identificar a los grupos de consumidores a los que merece la pena considerar como mercado meta.

Schiffman y Lazar (2010) conceptualizan a la segmentación del mercado como el proceso mediante el cual el mercado se divide en subconjuntos de consumidores con necesidades o características comunes.

Kolter y Keller (Kotler \& Keller, 2012) señalan que la psicografía es la ciencia que utiliza la psicología y la demografía para entender mejor a los consumidores. En la segmentación psicográfica los compradores se dividen en diferentes grupos con base en sus características psicológicas/de personalidad, su estilo de vida o sus valores. Las personas de un mismo grupo demográfico pueden exhibir perfiles psicográficos muy diferentes.

Un estudio psicográfico incluye una batería de enunciados seleccionados de un inventario psicográfico y por lo común se acompaña de escalas Likert, sobre las cuales se pide a los entrevistados que indiquen su nivel de acuerdo o desacuerdo con cada afirmación. Algunos de los factores que se examinan son parecidos a los rasgos de la personalidad; en tanto que otros incluyen mediciones de los motivos para comprar, las actitudes, las creencias y los valores. A causa de su versatilidad, los datos psicográficos se utilizan ampliamente en la segmentación y forman parte de casi cualquier esquema de segmentación híbrida (Schiffman \& Lazar Kanuk, 2010). 
Considerando la persistencia de los valores culturales fundamentales, son mucho los individuos que siguen creyendo en el trabajo, en el matrimonio, en la beneficencia y en la honestidad. Los valores fundamentales y las creencias pasan de padres a hijos, y son reforzados en las instituciones sociales (Kotler \& Keller, 2012).

Los valores personales proporcionan información de las aspiraciones y metas que las personas evalúan como más importantes, de ahí que permitan conocer y determinar las motivaciones que influyen en sus decisiones de compra (Kahle , Beatty, \& Homer, 1986).

La metodología del Rokeach Value Survey R.V.S. analiza la incidencia de los valores sobre el comportamiento y los vínculos con la clase social, edad, raza, religión y subculturas) (Rokeach, 1973) citado por (González Fernández, 2000). Se trata de una orientación práctica y operacional que confiere a estas variables una aplicación interdisciplinaria en el ámbito del Marketing.

Uno de los sistemas de clasificación basado en mediciones psicográficas más populares y comercialmente disponibles es el marco VALSTM de Strategic Business Insight (SBI). VALS, que significa "values and lifestyles" (valores y estilos de vida), clasifica a los adultos estadounidenses en ocho grupos principales (Kotler \& Keller, 2012).

La lista de valores (LOV) es un método desarrollado por el centro de investigación de encuestas de la Universidad de Michigan y tiene su origen en los estudios sobre valores realizados por Maslow, Rokeach y Feather. Se trata de una propuesta metodológica vinculada a la teoría de la adaptación social y considera que los individuos se adaptan a ciertos papeles o roles en la vida debido, entre otros factores a sus valores. La metodología LOV, utiliza 9 de los 36 ítems introducidos por Rokeach y se orientan fundamentalmente hacia la persona (Ramos Soler, 2008).

L.O.V. es un instrumento de medición abreviado que incluye parte de los valores terminales de ROKEACH, aportando una solución a la dificultad de ordenar jerárquicamente los dos grupos de 18 valores de dicha metodología. Utiliza una lista reducida de nueve valores (1) simplificando notablemente la tarea de clasificación: autoestima, seguridad, relaciones de afecto con los demás, sentido del cumplimiento, autorrealización, sentido de posesión, respeto a los demás, diversión y disfrute de la vida y, entusiasmo ( Kahle , Beatty, \& Homer, 1986) citado por González Fernández (González Fernández, 2000).

Para Merino Saénz (2009), al comparar las tres escalas: La Lista de Valores LOV, Valores y Estilos de de vida VALS y el Sistema de Valores de Rokeach RVS; los resultados de investigaciones indican que el método LOV tiene una mayor utilidad predictiva que el VALS en cuanto a tendencias del comportamiento del consumidor. Otra ventaja de de LOV es 
que se pueden analizar las variables sociodemográfica por separados, asi como la simplicidad de la encuesta. La metodologia LOV describe de una manera más amplia la vida diaria de la gente que el RVS, siendo además más simple de administrar debido a que se tienen que dar respuesta a un número de valores mas pequeño que en el RVS.

Respecto al interés del tema, en el contexto científico, existen trabajos previos que analizan los valores de los consumidores:

Irene Ramos Soler (2008) en la provincia de Alicante lleva a cabo un estudio con una muestra de 528 personas mayores de 65 años. Los resultados obtenidos dan una información muy positiva acerca de la fiabilidad y validez de la escala de valores LOV adaptada por primera vez a las personas mayores de 65 años. Reconociendo seis estilos de vida diferentes en la población objeto de estudio.

Otro estudio aplicando los valores en la segmentación del Mercado de turismo, es el realizado en la Universidad de Leon España en el año 2000. Una de sus aportaciones es el constrastar el constructo de estilo de vida medido mediante las variables de Actividades, Interes y opinions (A.I.O) está relacionado directamente con el Sistema de valores personales (LOV) así como con el comportamiento turístico del individuo (Gonzalez Fernández, Bello Acebrón, \& Muñiz Martínez, 2000).

\section{Metodología}

Se realiza un estudio cuantitativo- descriptivo transversal, desde la perspectiva conceptual de la Escala de Lista de Valores (LOV) atribuido a Khale (Ramos Soler, 2008). llevando a cabo un análisis comparativo en porcentajes de las respuestas con el programa de IBM SPSS .

Muestra: En este estudio se utiliza el muestreo por estratificación, considerando la variable edad, se entrevistaron a 1,000 personas de la ciudad de Saltillo, Coahuila, determinando que 261 sujetos que pertenecen a la "Generación Y" nacidos entre los años 1979 y 1994.

\section{Instrumento de Medición}

Para el presente estudio se utiliza la escala L.O.V La Lista de Valores y está compuesta de nueve variables:

1. Sentido de Posesión: Ser aceptado y necesitado por otros (amigos, familia...). Maslow postula que las necesidades sociales o de pertenencia/ de aceptación social se refiere a la pertenencia a un grupo, el ser aceptado por los compañeros, tener amistades, dar y recibir estima (Rogers \& Maslow, 2009). Tener cosas propias (Ramos Soler, 2008). 
2. Entusiasmo: Ganas de experimentar emociones y sentimientos. La Real Academia Española cita que el entusiasmo es la Exaltación y Fogosidad del ánimo, excitado por algo que lo admire o cautive. (RAE) Vivir con entusiasmo (Ramos Soler, 2008)

3. Relaciones de Afecto Con los Demás: Mantener relaciones positivas con otras personas incluyendo en un sentido de intimidad (Marriner Tomey Ana, 2007) Querer y que me quieran otras personas (Ramos Soler , 2008).

4. Autorrealización: Estar agusto y ser feliz con lo que uno hace o trabaja. La Real Academia Española señala que es la Consecución satisfactoria de las aspiraciones personales por medios propios (RAE). Sentirme a gusto conmigo mismo (Ramos Soler, 2008).

5. Respeto a los demás: Atención, acatamiento que se hace a alguien (RAE).

6. Placer y disfrute de la vida.

7. Seguridad: Estar seguro y protegido de desgracias. Sentirme seguro (Ramos Soler, 2008).

8. Autoestima: Tener buena imagen de uno mismo (Ramos Soler, 2008).

9. Sentido del Cumplimiento: Importancia de hacer bien las cosas. Tener éxito en lo que uno quiere hacer. Sentir la satisfacción del deber cumplido (Ramos Soler, 2008).

Escala Adaptada de Sarabia, De Juan y González Fernández (2009).

Para el trabajo de campo se utiliza el cuestionario en el que se pide a los individuos que ordenen jerárquicamente los valores que se muestran o que los evalúe según una escala de 9 puntos e identifique los dos que considere más importantes. El cuestionario se diseñó con una escala del 1 al 9 tipo Likert, en donde 9 significa "extremadamente importante" y 1 "en absoluto importante".

\section{Resultados}

Resultados del Estudio Empírico. Análisis descriptivo.

\section{Perfil de los Entrevistados}

Los resultados obtenidos al aplicar el instrumento a un grupo de 261 personas de la Generación "Y" en la ciudad de Saltillo Coahuila, respecto a los factores sociodemográficos, presentan resultados preliminares siendo los más relevantes los siguientes: Los millenials sus edades oscilan entre 21 y 35 años; el 51.7\% de los entrevistados son hombres y el $48.3 \%$ 
son mujeres; el $66.7 \%$ cuenta con estudios de Licenciatura y el $18.4 \%$ bachillerato; en relación con el estado civil el 67.8\% manifiesta ser soltero.

Se lleva a cabo un análisis comparativo en porcentajes con el programa IBM SPSS, encontrándose que el valor más importante ubicado en primer lugar es: Autorrealización y en segundo lugar el Autoestima.

\section{Análisis Ji cuadrado de Pearson Hipótesis}

H0: Sentido de posesión es independiente del género.

H1: Hay relación entre el género y Sentido de Posesión.

H1: No existen diferencias significativas entre hombres y mujeres con respecto a Sentido de Posesión.

Tabla 1. Ji cuadrado de Pearson

\begin{tabular}{llllllllllll}
\hline & & \multicolumn{1}{c}{ Sentido de posesión } & \multicolumn{4}{c}{ Total } \\
\cline { 2 - 12 } & & 1 & 2 & 3 & 4 & 5 & 6 & 7 & 8 & 9 & \\
\hline \multirow{2}{*}{ Genero } & HOMBRE & 6 & 3 & 6 & 6 & 14 & 27 & 40 & 23 & 10 & 135 \\
\cline { 2 - 11 } & MUJER & 6 & 4 & 12 & 10 & 17 & 17 & 15 & 35 & 10 & 126 \\
\hline \multirow{2}{*}{ Total } & 12 & 7 & 18 & 16 & 31 & 44 & 55 & 58 & 20 & 261
\end{tabular}

Pruebas de chi-cuadrado

\begin{tabular}{llll}
\hline & Valor & gl & $\begin{array}{l}\text { Sig. asintótica } \\
\text { (bilateral) }\end{array}$ \\
\hline Chi-cuadrado de Pearson & $19.265^{\mathrm{a}}$ & 8 & .014 \\
\hline Razón de verosimilitudes & 19.758 & 8 & .011 \\
\hline Asociación lineal por lineal & .964 & 1 & .326 \\
\hline N de casos válidos & 261 & & \\
\hline $\begin{array}{l}\text { a. } 2 \text { casillas (11.1\%) tienen una frecuencia esperada inferior a 5. La } \\
\text { frecuencia mínima esperada es 3.38. }\end{array}$
\end{tabular}

Fuente: Elaboración Propia

En las Tablas 1, y $1^{\text {a }}$ se encuentran los resultados de la ji cuadrado de Pearson que muestra una significancia > a.05, no se rechaza la $\mathrm{H} 0$, por lo que se puede afirmar con una confianza al $95 \%$ que no hay diferencias significativas entre hombres y mujeres con respecto al sentido de posesión. 
Tabla 2. Valor \#1 más importante y el Género

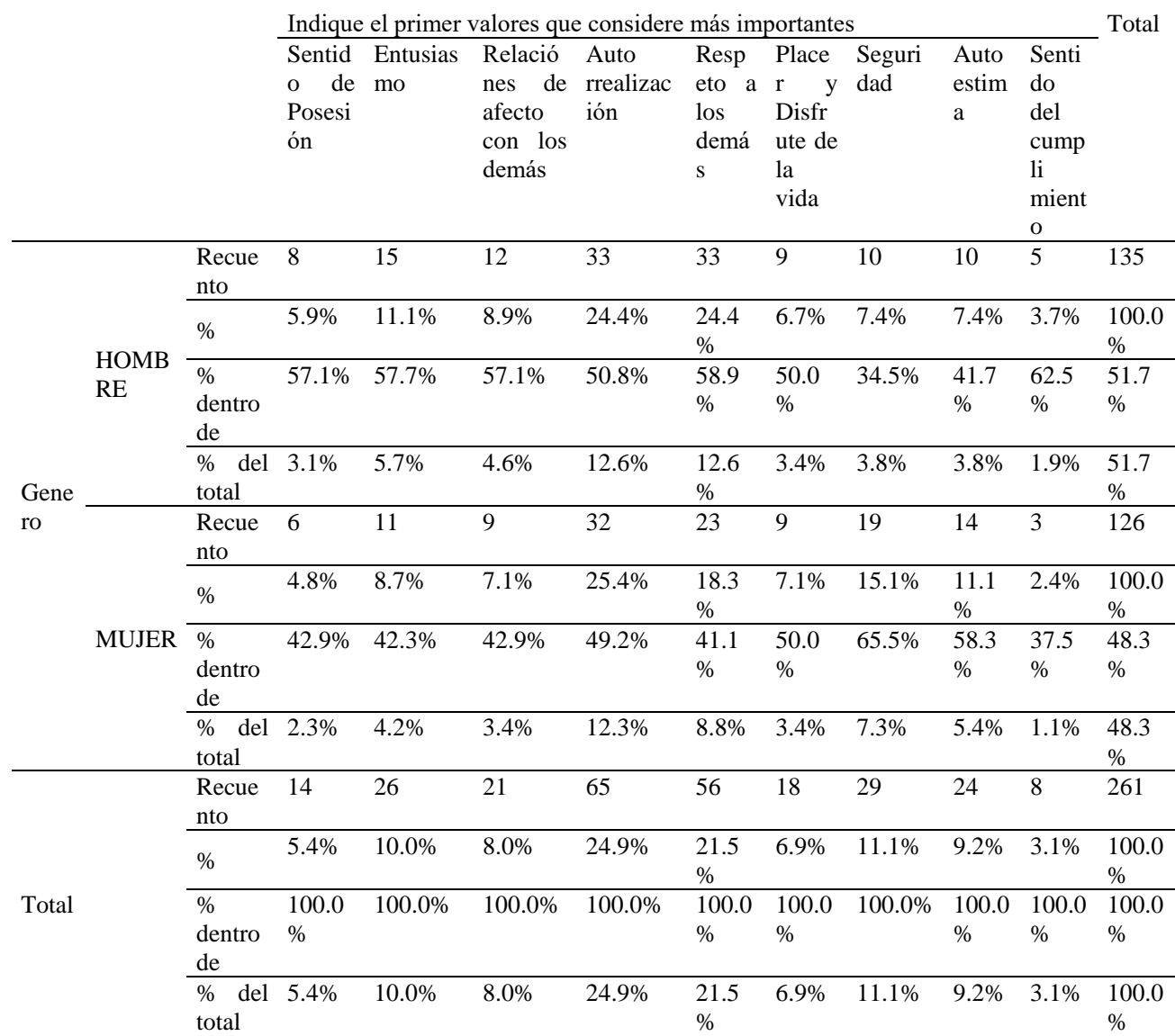

Fuente: Elaboración Propia

En la Tabla 2 se encontró que los porcentajes de la variable Valor \#1 mas importante" porcentajes del 3.1\%. para los hombres y el 2.3\% para las mujeres en la respuesta correspondiente a "Sentido de posesión", en la respuesta "Entusiasmo" los porcentajes representaron un 5.7\% en los hombres, mientras que para las mujeres fue un $4.2 \%$; en la variable Relaciones de afecto con los demás se obtuvo un $8 \% 4.6$ los hombres y $3.4 \%$ las mujeres; siendo la respuesta "Autorrealización" la que más porcentaje representó para los hombres con un $12.6 \%$ y $12.3 \%$ para las mujeres, con un total de $24.9 \%$; en segundo lugar lo ocupó la respuesta Respeto a los demás con un $21,5 \% 12.6 \%$ los hombres y $8.8 \%$ las mujeres; a la variable placer y disfrute de la vida le corresponde un 6.9\%, la variable Seguridad ocupó el tercer sitio con un 11\%, Autoestima con el 9.2\% y sentido del cumplimiento un $3.1 \%$. 
Tabla 3. Valor \#2 de importancia y el Género

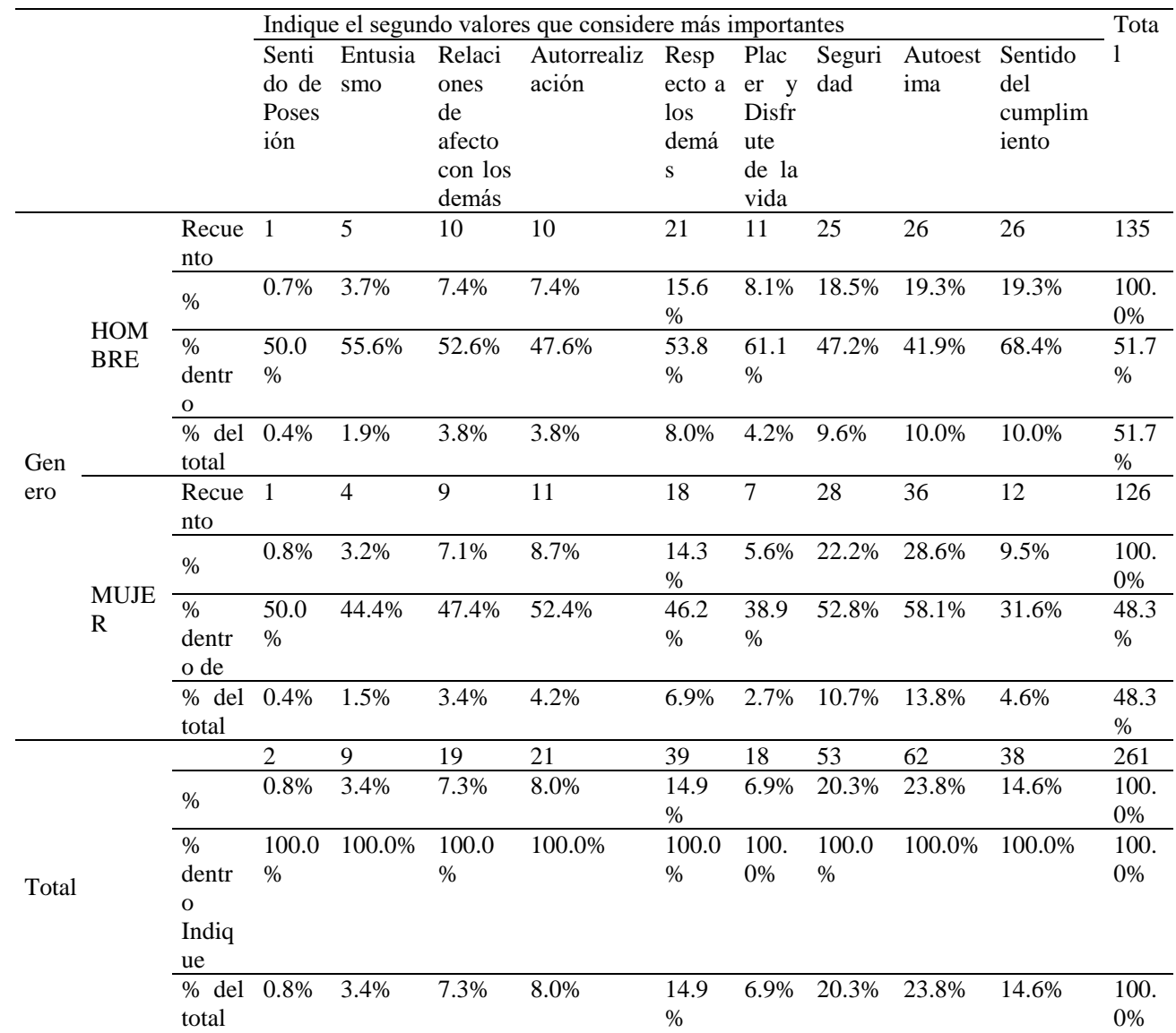

Fuente: Elaboración Propia

En la Tabla 4 al cuestionarles su valor número dos en importancia, el $23.8 \%$ le otorgó la calificación más alta a la variable Autoestima, con un $10 \%$ el sexo masculino y con $13.8 \%$ el sexo femenino; siguiendo con un $20.3 \%$ el ítem Seguridad correspondiendo a un $9.6 \%$ los hombres y las mujeres con un $10.7 \%$; la variable Respeto a los demás es el tercer ítem con un $14.9 \%$.

\section{Conclusión}

Al inicio del artículo se expresa el interés por la investigación del consumidor. El objetivo del presente trabajo es la aportación con evidencia empirica en el estudio de la mercadotecnia.

Los resultados obtenidos al realizar un análisis comparativo en porcentajes con el programa IBM SPSS, ofrecen un primer diagnóstico: El valor más importante para los millennials saltillenses es la Autorrealización, es decir, sentirse agusto con ellos mismos. Y en segundo lugar, un valor muy 
parecido al anterior, el Autoestima, que es el tener buena imagen de ellos mismos. Referente al valor Respeto a los demás manifestado como importante en tercer lugar para los entrevistados, se coincide con Kotler y Keller (2012), quienes afirman que los valores fundamentales y las creencias pasan de padres a hijos, y son reforzados en las instituciones sociales.

Otro hallazgo encontrado en el análisis estadístico es que no existen diferencias significativas entre los valores de hombres y mujeres. Y con base en los resultados, podemos concluir que un diagnóstico como el presentado hasta aquí nos permite identificar los elementos claves para establecer estrategias de mercadotecnia en los productos y servicios que eleven la autoestima y hagan sentir agusto a los clientes, como lo son: la ropa, el gimnasio, los viajes, la belleza, salud, entre otros.

Una característica importante en este estudio es que el estilo de vida es personal y cambia continuamente. El estudiar los estilos de vida y los cambios de valores de los jóvenes coadyuva a la detección de necesidades latentes, función principal de la mercadotecnia para la atención de manera eficaz a los Mercados metas. Una de las limitantes del estudio es el tamaño de la muestra, por lo que los resultados no pueden generalizarse. Se pretende continuar con los análisis comparativos con otras generaciones y ampliar la muestra a otros estados del país. Cabe destacar que el estudio es un Proyecto de la Red Nacional de Investigación "Gestión de la Mercadotecnia del Consorcio de Universidades Mexicanas".

\section{References:}

1. Kahle , L. R., Beatty, S. E., \& Homer, P. (1986). Alternative Measurement Approaches to Consumer Values: The List of Values (LOV) and Values and Life Style (VALS). Journal of Consumer Research, 405-409.

2. Schiffman, L. G., \& Lazar Kanuk, L. (2010). Comportamiento del Consumidor (DÉCIMA EDICIÓN ed.). México: PEARSON EDUCACIÓN.

3. González Fernández, A. M. (2000). "Los valores personales en el comportamiento del consumidor. Revisión de diversas metodologías aplicadas al marketing ". ESIC Marketing, 9 -36.

4. Gonzalez Fernández, A., Bello Acebrón, L., \& Muñiz Martínez, N. (2000). Orienación de la empresa turística del siglo XXI a los valores personales y los estilos de vida de los turistas. Cuadernos UMA ES.

5. Hair, J. F., E., A. R., Tatham, R. L., \& Black, W. C. (1999). Análisis Multivariante (quinta Edición). Madrid, España.: Pearson Educación. 
6. Hernández, S. R., Fernández, C. C., \& Baptista, L. P. (2014). Metodología de la Investigación (Sexta Edición). México: McGrawHill.

7. Kotler, P., \& Keller, k. (2012). Dirección de Marketing. México: Pearson Educación.

8. Marriner Tomey Ana, R. A. (2007). Modelo de promoción de la salud: Nola Pender. Modelos y Teorías en Enfermería. La Habana, Cuba.: 4ta edición .Editorial Ciencias Medicas.

9. Merino Saénz, M. J. (2009). Inmigración y consumo. Estilos de vida de los inmigrantes en España. Madrid: ESIC Editorial.

10. O'Shaughenessy, J., \& O'Shaughenenessy, N. (2002). "Marketing, the Consumer Society and Hedonism". European Journal of Marketing, 36, 524-548.

11. RAE. (s.f.). Real Academia Española. Obtenido de http://dle.rae.es/srv/search?m=30\&w=entusiasmo

12. Ramos Soler, I. (2008). Estilo de vida y valores en las personas mayores de 65 años. Metodología de Encuestas .

13. Rogers, C., \& Maslow, A. (2009). La psicologia humanista. Obtenido de Academia Edu.

14. Rokeach, M. (1973). The Nature of Human Values. New York: free Press.

15. Sarabia Sánchez, F. J., De Juan Vigaray , M., \& Gonzalez Fernández, A. M. (2009). Valores y estilos de vida de los consumidores: como entenderlos y medirlos. Madrid: Pirámide.

16. Sprotles, G. B., \& Kendall, E. L. (1986). A Methodology for Profiling Consumers' Decision-Making Styles. Journal of Consumer Affairs, 267-279. 